\title{
High-contrast Fano resonances in single semiconductor nanorods
}

\author{
Diego R. Abujetas, ${ }^{\dagger}$ Miguel A. G. Mandujano, ${ }^{\ddagger}$ Eugenio R. Méndez, ${ }^{\ddagger}$ and José \\ A. Sánchez-Gil ${ }^{*, \dagger}$ \\ $\dagger$ Instituto de Estructura de la Materia, Consejo Superior de Investigaciones Científicas, \\ Serrano 121, 28006 Madrid, Spain \\ $\ddagger$ División de Física Aplicada, Centro de Investigación Científica y de Educación Superior \\ de Ensenada, Carretera Ensenada-Tijuana No. 3918, Ensenada, B.C. 22860, México \\ E-mail: j.sanchez@csic.es
}

\begin{abstract}
Fano resonances in plasmonics have received a widespread attention for their distinctly narrow asymmetric line shapes. A variety of configurations have been considered, requiring either complex metallic nanostructures or being extremely faint if originated in simple single nanoparticles. Here we report on the emergence of highcontrast, strongly asymmetric Fano line shapes in the light scattered from semiconductor nanorods. Numerical calculations are carried out for the scattering cross sections of finite semiconducting nanorods, with dimensions such that the lowest-order (transverse) Mie resonances coexist with the lowest-order guided modes. Such intense narrow Fano resonances are strongly/weakly asymmetric in TE/TM polarization, and overlap with the Mie-like background. A physical interpretation is presented stemming from the (strong or weak) interference of the far-field angular patterns of Mie resonances (indeed, of both magnetic and dielectric dipole character) with narrow Fabry-Perot
\end{abstract}


(guided mode) resonances, the latter calculated through a 1D line current model. A quasi-analytical expression is developed for the scattering cross sections that reproduce fairly well the Fano numerical line shapes, along with a generalized Fano formula, with fitting factors confirming their high asymmetry and contrast. These high-contrast, strongly asymmetric Fano resonances herein obtained could be potentially exploited in Nanophotonics and Sensing in the visible and near-IR, eased by simplified fabrication requirements of shape (nanorod) and material (semiconductor).

\section{Keywords}

Fano resonance, magnetic light, semiconductor nanorod, Fabry-Perot waveguide modes, Mie scattering

High-refractive index nanophotonic structures have recently emerged as promising candidates to tailor light at the nanoscale. ${ }^{1,2}$ This interest was triggered by the strong magnetic dipole resonances found in the visible spectral range for semiconductor nanoparticles, ${ }^{3-5}$ simply explained through Mie theory for spherical subwavelength particles with high-refractive index. ${ }^{6}$ The emergence of such magnetic dipole resonances was experimentally verified later on, ${ }^{7-9}$ thereby coining the term "magnetic light". ${ }^{8}$ Unlike plasmonics, which suffers from high losses and fabrication issues, resonant dielectric nanostructures are nearly lossless, precluding energy dissipation into heat, ${ }^{10}$ and allow for an all-dielectric fabrication engineering. Therefore, in light of their unique optically induced magnetic and (also) electric resonances, which may indeed coexist, high-index dielectric nanostructures are emerging as a new field in nanophotonics, with the potential: to improve, or at least complement, plasmonics in many potential applications; and to provide indeed novel functionalities at the nanoscale. Thus far, magnetic resonances have been shown to manifest a variety of fascinating phenomenology such as: nanoantenna directionality, ${ }^{7,11-14}$ Raman scattering, ${ }^{10}$ metamaterials and metasurfaces with a variety of optical properties, ${ }^{15-22}$ enhanced nonlinear effects (including second-

and third-harmonic generation, and ultrafast all-optical switching), ${ }^{23-25}$ photoluminescence 
shaping, ${ }^{26}$ and optoelectronic devices. ${ }^{27-31}$

Among all that, the emergence of distinct Fano resonances in all-dielectric nanostructures remains largely unexplored. Only very recently some attention has been paid to Fano resonances in all-dielectric nanoparticles, nonetheless requiring either nanosphere dimers, oligomers, nanobelts, nanocrosses, or more complex arrangements. ${ }^{24,32-37}$ Fano resonances are asymmetric resonances resulting from the interference between a narrow (dark) resonance with a continuum, or a broad bright resonance, which have been intensively investigated at the nanoscale, ${ }^{38}$ and specially in plasmonics and metamaterials, ${ }^{39-41}$ due to their unique lineshapes that are highly promising for potential applications. ${ }^{39}$ Recall that, in general, very complex metal nanostructures are required to obtain remarkable Fano resonances; ${ }^{38,39}$ whilst those found in simple geometries such as single metallic nanostars ${ }^{42}$ or nanorods ${ }^{5,43-46}$ are relatively faint.

In this work we propose to use single semiconductor nanoparticles with simple finite cylindrical geometry, as in the latter case of metallic nanorods, which will be shown however to reveal a much richer phenomenology, indeed leading to strong and narrow Fano resonances. Note that semiconductor nanobelts and nanorods a few hundred nanometers long, to be distinguished from $>1 \mu \mathrm{m}$ nanowires, have been investigated to address some interesting scattering properties, in connection with their shape dependence ${ }^{47-49}$ or with the transition of Mie resonances from a nanosphere to a short nanocylinder, ${ }^{50}$ nonetheless revealing no Fano line shapes; except for a low-contrast measured for . We first study the scattering efficiencies of semiconductor nanorods with diameter below $\sim 200 \mathrm{~nm}$, thus supporting a few guided modes while exhibiting lowest Mie resonances in the optical domain. It is shown that nanorod lengths of about half-micron ensure that low-order (narrow and intense) FabryPerot resonances of the supported guided modes overlap with the Mie resonances, leading to either strongly or weakly asymmetric (Fano) line shapes with a large contrast over the background. The corresponding higher or lower degree of interference is then explained through a theoretical model as a result of the far-field overlap of broad Mie modes with 
narrow Fabry-Perot resonances, leading to an heuristic quasi-analytical expression for the Fano line shapes that fully reproduces the numerical scattering cross sections, in agreement also with a generalized Fano formula.

\section{Scattering cross sections of semiconductor nanorods}

First, we present in Figure 1 numerical calculations of the scattering efficiency spectra $Q_{\text {sca }}$ of semiconductor nanorods with varying length $L$ and fixed radius $R=80 \mathrm{~nm}$, carried out through a free-software implementation, called SCUFF; ${ }^{51,52}$ the scattering geometry is depicted therein. The refractive index is fixed at $n=3.5$ corresponding to GaP at $\lambda=400$ $\mathrm{nm} ;{ }^{53}$ recall that, without absorptive losses, the extinction efficiency is identical to the scattering one $Q_{\text {ext }}=Q_{\text {sca }}$. For simplicity we have neglected the (slight) dispersion of the real part of the refractive index in the spectral range $\lambda=400-1000 \mathrm{~nm}$ to neatly determine the impact of nanorod dimensions on the spectral properties of all resonances; however, as long as absorption is negligible, ${ }^{14}$ our results could be applicable throughout the visible and near-infrared and to any other semiconductor with similar refractive index, such as cristalline $\mathrm{Si}^{21,22,47}$ or i.e. GaAs, InP, etc., upon scaling wavelength and nanorod dimensions accordingly if needed. The nanorod radius is fixed at $R=80 \mathrm{~nm}$ (shown below to be in the range of interest for coupling to few lowest-order guided modes), whereas the length is varied between $L=$ $400-1000 \mathrm{~nm}$. Indeed, since the refractive index is fixed, variations of the nanorod radius can be easily inferred from Figure 1, considering the corresponding $Q_{\text {sca }}$ for nanorod length $L$ such that the aspect ratio $L / R$ is preserved, with wavelength accordingly scaled. Larger aspect ratios lead to denser spectra with overlapping resonances; interestingly, as mentioned above, shorter nanorods with lower aspect ratios have been studied in a recent work with the different aim of describing the transition from spherical to cilindrical resonances. ${ }^{50}$

Basically, leaving aside broad dispersionless ( $L$-independent) resonances (to be associated to lowest-order Mie resonances of the infinite cylinder), a few narrow resonances appear for 

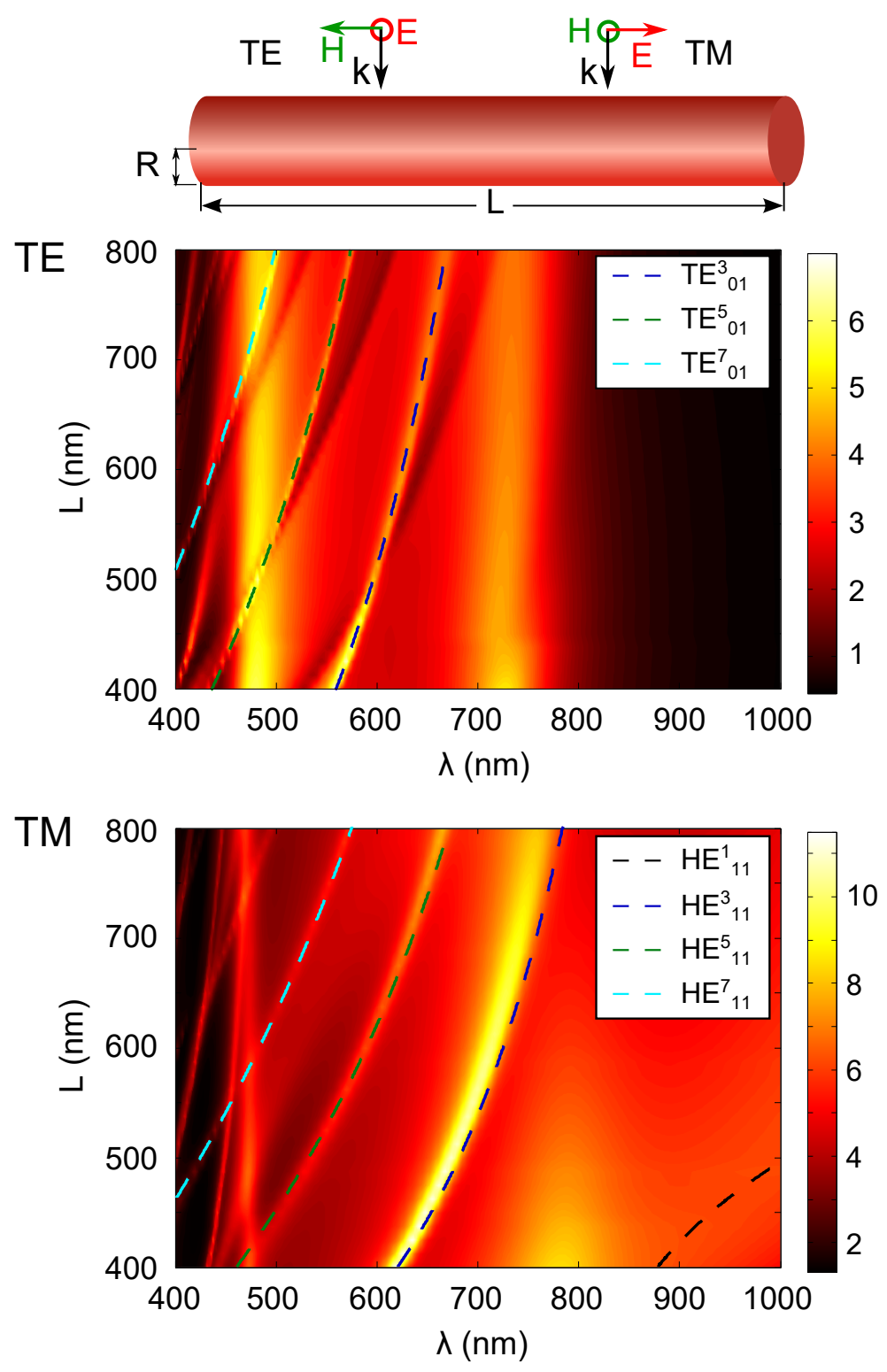

Figure 1: Color maps of the spectral dependence of the scattering efficiency $Q_{\text {sca }}$ for a finite semiconductor $(n=3.5)$ nanowire of radius $R=80 \mathrm{~nm}$ and normal incidence, numerically calculated (SCUFF, see Methods) for varying length $L$ : (a) TE and (b) TM polarization, separately. The condition for Fabry-Perot resonances of the corresponding guided modes from eq 1 is superimposed (colored curves). The schematic reveals the scattering geometry. 
both polarization that redshift for increasing nanorod length crossing in different ways the broader resonances. Essentially, we assign them to Fabry-Perot resonances occurring at multiples of half-wavelength of the guided modes supported by the semiconductor nanorod, identical to those involved in nanowire photoluminescence. ${ }^{54}$ The resonance condition is thus described by:

$$
k_{z}(\omega) L^{*}=\pi l, l=1,2,3, \ldots
$$

$k_{z}$ being the guided mode complex wavevector along the cylinder axis, $\omega / c=2 \pi / \lambda$ ( $\lambda$ is the wavelength in vacuum), and $L^{*}$ an effective wavelength very close to $L$ that depends on the guided mode involved, ${ }^{54}$ as explained below. $k_{z}(\omega)$ itself is frequency dependent, and obeys the following dispersion relation ${ }^{55}$

$$
\begin{array}{r}
{\left[\frac{\mu_{c}}{u} \frac{J_{m}^{\prime}(u)}{J_{m}(u)}-\frac{\mu}{v} \frac{H_{m}^{\prime}(v)}{H_{m}(v)}\right]\left[\frac{\epsilon_{c}}{u} \frac{J_{m}^{\prime}(u)}{J_{m}(u)}-\frac{\epsilon}{v} \frac{H_{m}^{\prime}(v)}{H_{m}(v)}\right]} \\
=m^{2} \frac{\left(k_{z} R\right)^{2}}{(\omega R / c)^{2}}\left(\frac{1}{v^{2}}-\frac{1}{u^{2}}\right)^{2}
\end{array}
$$

where $u=k_{c} R, v=k R, m$ is an integer, and $J_{m}$ and $H_{m}$ standard (first-kind) Bessel and Hankel functions; the transverse components of the mode wavevector at frequency $\omega$, inside $\left(k_{c}\right)$ and outside $(k)$ the cylinder, are

$$
\begin{gathered}
k_{c}^{2}=\epsilon \frac{\omega^{2}}{c^{2}}-k_{z}^{2}, \\
k^{2}=\frac{\omega^{2}}{c^{2}}-k_{z}^{2},
\end{gathered}
$$

where the nanorod dielectric permittivity $\epsilon$ is related to the corresponding refractive index through $n=(\epsilon \mu)^{1 / 2}$ (assuming also that the embedding medium is vacuum, $n=1$, and nonmagnetic cylinder, $\mu=1$ ). For our purpose, only guided modes with $k_{z}>\omega / c$ will be considered, propagating along the nanorod, and bouncing back and forth so that Fabry- 
Perot resonances occur. Only the three lowest-order guided modes play a role for the nanorod dimensions and spectral region shown above: the no cutoff, lowest order hybrid $\mathrm{HE}_{11}$ mode, and the pure transverse $\mathrm{TM}_{01}$ and $\mathrm{TE}_{01}$ modes.

The corresponding FP resonances from eqs 1,2 are tagged in Figure 1 through a superscript indicating the $l$-th FP-order in eqs 1 . Note that no FP resonances associated to the $\mathrm{TM}_{01}$ are indicated. Indeed, the frequency above which this mode becomes guided is the same as that of the $\mathrm{TE}_{01}$ mode, but it is weakly guided with a small wavevector $k_{z} \gtrsim \omega / c$ (large wavelength) so that no significant FP are excited within the $\omega, L$ domain in Figure $1 \mathrm{~b}$. For TE polarization, most $\mathrm{FP}$ resonances stem from the $\mathrm{TE}_{01}$ mode, as expected due to symmetry. This mode has a complex reflection coefficient, which we account for by assuming that the effective length in eq 1 is slightly different from the nanorod length $L^{*}=1.05 L$ (cf. Ref $\left.{ }^{54}\right)$. The agreement of the FP condition for $\operatorname{TE}_{01}^{(l)}(l=1,3,5,7, \ldots)$ with the resonances in Figure 1a is excellent (recall no even resonances are present at normal incidence in Figure 2). Likewise, very good agreement is found in TM polarization for FP resonances $\mathrm{HE}_{11}^{(l)}$ $(l=1,3,5,7, \ldots)$, for which the reflection coefficient is real, ${ }^{54}$ so that $L^{*}=L$. Low-order FP resonances associated to higher-energy guided modes appear in the high-frequency regime too.

In addition to those narrow FP resonances, a background pattern with various broad resonances nearly dispersionless is observed in Figure 1. To properly characterize them, we present the numerical calculations of $Q_{\text {sca }}$ for a fixed nanorod length $L$ in Figures 2 and 3 ; the Mie-like scattering efficiencies $Q_{\text {Mie }}$ are also included for normal incidence with respect to the nanorod axis, calculated through the following expressions ${ }^{6}$

$$
\begin{aligned}
& Q_{\text {Mie }}^{(T E)}=\frac{2}{k R}\left[\left|a_{0}\right|^{2}+2 \sum_{m=1}^{\infty}\left|a_{m}\right|^{2}\right], \\
& Q_{\text {Mie }}^{(T M)}=\frac{2}{k R}\left[\left|b_{0}\right|^{2}+2 \sum_{m=1}^{\infty}\left|b_{m}\right|^{2}\right],
\end{aligned}
$$

where $a_{0}, a_{m}, b_{0}, b_{m}$ are the corresponding Mie scattering coefficients, ${ }^{6}$ for a plane wave im- 


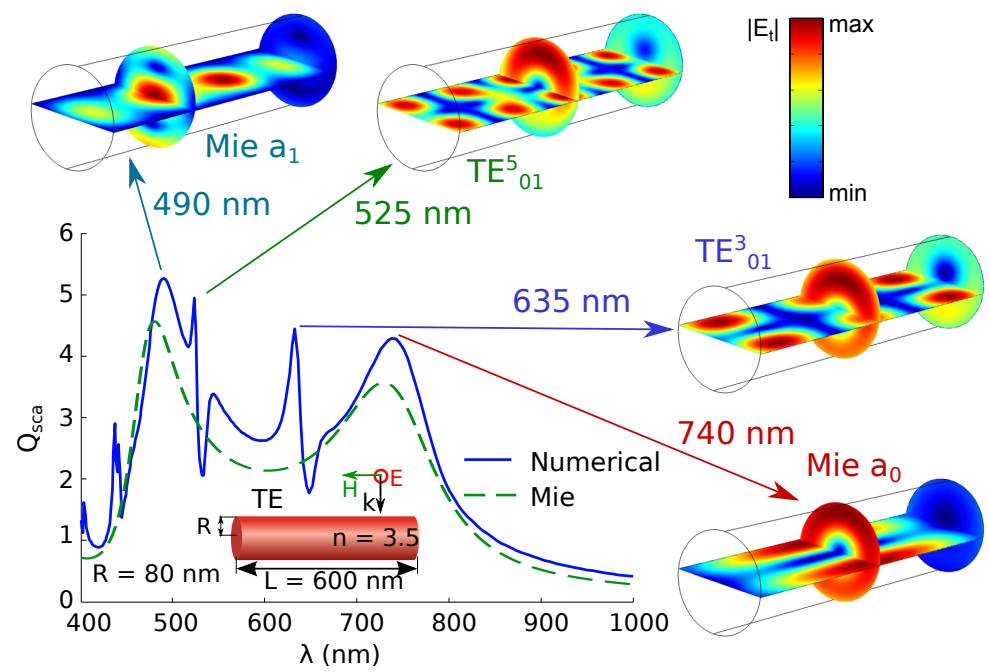

Figure 2: Spectral dependence of the scattering efficiency $Q_{\text {sca }}$ (blue solid curve) at normal incidence (TE polarization) for a finite semiconductor $(n=3.5)$ nanorod of radius $R=80$ $\mathrm{nm}$ and fixed length $L=600 \mathrm{~nm}$, numerically calculated (SCUFF). Mie calculation for an infinite cylinder with the same radius is also included (green dashed curve). Contour color maps are also included at various fixed wavelengths corresponding to Mie and FP resonances, showing the transverse electric field amplitude $\left|E_{t}\right|=\left(\left|E_{r}\right|^{2}+\left|E_{\phi}\right|^{2}\right)^{1 / 2}$ on the longitudinal and (two) transverse cross sections of the nanorod.

pinging perpendicular to the infinite nanocylinder, polarized either perpendicular (TE) or parallel (TM) to its axis.

For TE polarization (see Figure 2), three narrow resonances are clearly identified, two of them at least revealing a strong asymmetric lineshape, standing over broader resonances. Those two broad resonances centered at $\lambda=740$ and $490 \mathrm{~nm}$, also observed in the $Q_{\text {Mie }}$, obviously correspond to the two lowest-order Mie resonances stemming from Mie coefficients $a_{0}$ and $a_{1} ; a_{0}$ can be considered an effective longitudinal magnetic dipole (eMD), and $a_{1}$ is the transverse electric dipole (ED) contribution. This is in turn confirmed by the transverse electric field patterns inside the nanorod numerically calculated at $\lambda=740,490 \mathrm{~nm}$ through FEM simulations (COMSOL, see Methods), also included in Figure 2. Near-field maps inside the nanorod are also shown at the narrow Fano resonances for $\lambda=635 \mathrm{~nm}$ and $525 \mathrm{~nm}$ to guide their assignment: both of them correspond to the $\mathrm{TE}_{01}$ guided mode predicted by the FP condition (eq 1 ) with $l=1,3$, as confirmed through the following arguments. The transverse electric field maps exhibit a ring shape with mostly azimuthal polarization; the 


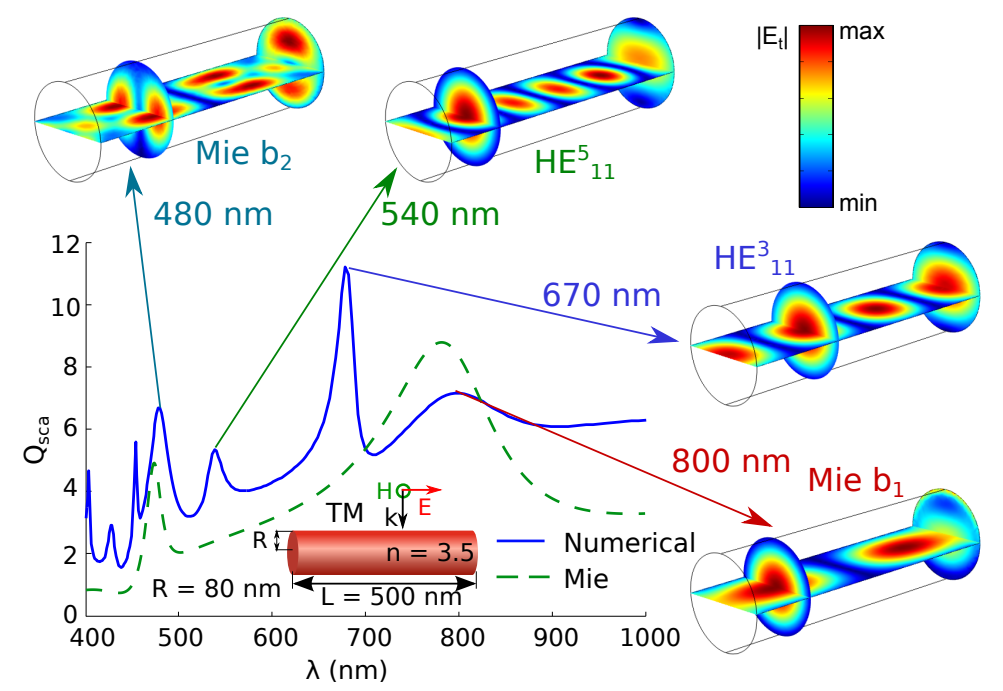

Figure 3: Spectral dependence of the scattering efficiency $Q_{\text {sca }}$ (blue solid curve) at normal incidence and TM polarization for a finite semiconductor $(n=3.5)$ nanorod of radius $R=80$ $\mathrm{nm}$ and fixed length $L=500 \mathrm{~nm}$, numerically calculated (SCUFF). Mie calculation for an infinite cylinder with the same radius is also included (green dashed curve). Contour color maps are also included at various fixed wavelengths corresponding to Mie and FP resonances, showing the transverse electric field amplitude $\left|E_{t}\right|=\left(\left|E_{r}\right|^{2}+\left|E_{\phi}\right|^{2}\right)^{1 / 2}$ on the longitudinal and (two) transverse cross sections of the nanorod.

transverse magnetic field, not shown, is dominated by its axial component. Both features unequivocally associated to the $\mathrm{TE}_{01}$ guided mode of the infinitely long cylinder, ${ }^{54}$ as confirmed by the ring-shaped, in-plane electric field pattern with rotating polarization depicted in Figure 4a for $\mathrm{TE}_{01}$ guided mode at $\lambda=635 \mathrm{~nm}$ (the one at $\lambda=525 \mathrm{~nm}$, nearly identical, is not shown). On the other hand, the number of nodes observed in the near-field maps along the nanorod length agrees well with the expected $\mathrm{FP}$ order: $l=3,5$, respectively, for the Fano resonances at $\lambda=635$ and $525 \mathrm{~nm}$.

Before describing in detail the asymmetry of such strong Fano lineshapes, let us now discuss the TM polarization in Figure 3. Note that similar narrow resonances are identified over the broad Mie-like resonances. In this case, broad Mie resonances stem from $b_{0}, b_{1}, b_{2}$ coefficients with, respectively, effective longitudinal electric dipole (eED), and transverse magnetic dipole (MD) \& quadrupole character; the lowest-order $b_{0}$ term being extremely broad and lying at lower frequencies not shown in Figure 3 (incidentally, it can be observed in Figure $1 \mathrm{~b}$ at $\lambda \gtrsim 1 \mu \mathrm{m})$. Narrow resonances at $\lambda=670 \mathrm{~nm}$ and $540 \mathrm{~nm}$ stem both from the 

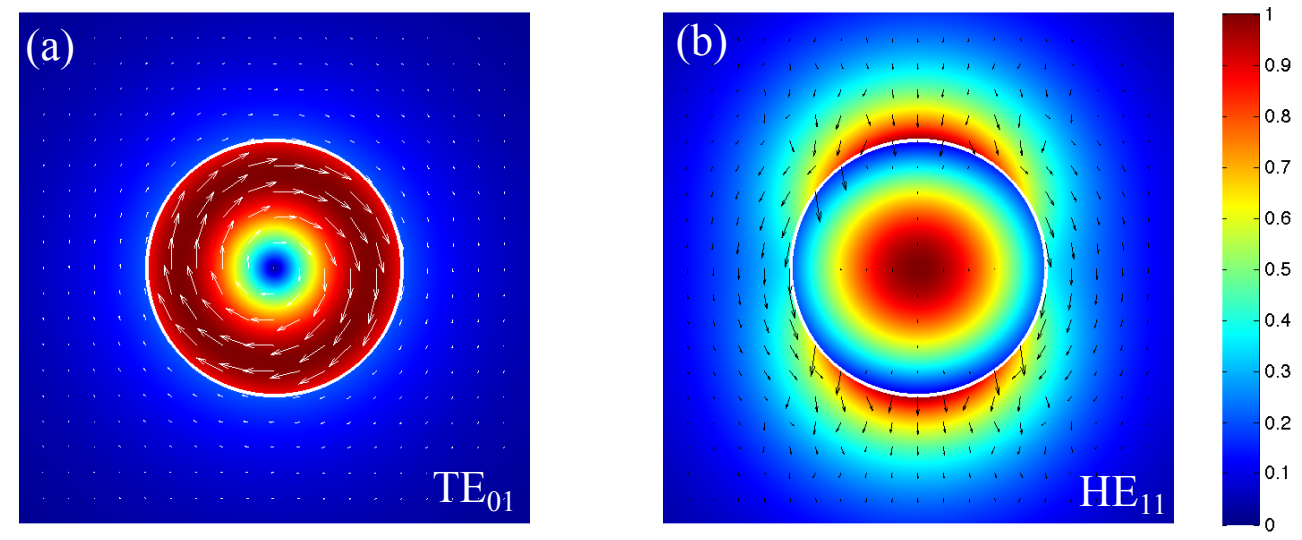

Figure 4: Color maps of the electric field norm of guided modes supported by an infinite semiconductor $(l=3.5)$ cylinder of radius $R=80 \mathrm{~nm}$ at the following wavelengths: (a) $\mathrm{TE}_{01}$ mode at $\lambda=635 \mathrm{~nm}$, and (b) $\mathrm{HE}_{11}$ mode at $\lambda=670 \mathrm{~nm}$. The arrows represent the direction of the in-plane components of the electric field, with size proportional to the norm.

$l=3,5 \mathrm{FP}$ orders of the $\mathrm{HE}_{11}$ guided mode, as determined by the $\mathrm{FP}$ condition 1 . Indeed, the electric field maps inside the nanorod (transverse centered lobe with hybrid polarization), also included, agree very well with the expected in-plane electric field pattern of the $\mathrm{HE}_{11}$ guided mode at $\lambda=670 \mathrm{~nm}$ for an infinite cylinder (see Figure $4 \mathrm{~b}$ ).

However, such narrow resonances exhibit a weakly asymmetric line shapes, much unlike the highly asymmetric Fano resonances in TE polarization described above, even though some of the modes involved in both kind of resonances have identical FP orders; recall that FP order has been associated to the parity order used to describe Fano/Lorentz line shapes in metallic nanorods. ${ }^{46}$ Therefore, further investigation is needed to account for this richer phenomenology found for semiconductor, rather than metal, nanorods supporting a wide variety of both broad and narrow (high-contrast) resonances. Incidentally, additional calculations are provided in the Supporting Information, Figure S1, for a real material such as $\mathrm{GaP}$ with proper dielectric function dispersion ${ }^{53}$ taken into account, for the same nanorod dimensions used in Figures 2 and 3. All relevant features are reproduced with slight spectral displacements induced by (real part) refractive index variations; particularly, very similar high contrast Fano resonances are obtained. As expected, absorption dominates for wavelengths smaller than the GaP band gap $(\lambda \lesssim 480 \mathrm{~nm})$ and smooths out any narrow resonance. 


\section{Interplay of Fabry-Perot resonances and Mie modes}

Therefore, to fully understand the emergence of Fano resonant modes for semiconductor nanorods, we propose a simple model combining (transverse) Mie scattering resonances (playing the role of the broad continuum) and (longitudinal) FP resonances (dark modes).Nearfield coupling between modes within a single nanorod is rather complex. This was shown in the case of metal nanorods, ${ }^{44}$ where the near-field pattern of the narrow resonant mode predominates within the spectral region of the Fano line shape; likewise, the near-field patterns at resonances in Figures 2 and 3 are dominated by the FP resonances. Hence, we assume that the interference is more clearly manifested in the far field, expressing the scattering cross section in the following manner:

$$
\operatorname{SCS}(\omega)=\int d \Omega \frac{\Re\left\{\mathbf{E}_{\Omega}(\mathbf{r}, \omega) \times \mathbf{H}^{*}(\mathbf{r}, \omega)\right\}}{2\left|E_{0}\right|^{2}},
$$

where the angular- and frequency-dependent far-field pattern $\mathbf{E}_{\Omega}(\mathbf{r}, \omega)$ has two contributions:

$$
\mathbf{E}_{\Omega}(\mathbf{r}, \omega)=\mathbf{E}^{(\mathrm{Mie})}(\mathbf{r}, \omega)+\mathbf{E}^{(\mathrm{FP})}(\mathbf{r}, \omega),
$$

stemming from transverse Mie-like scattering $\mathbf{E}^{(\mathrm{Mie})}$ and the longitudinal Fabry-Perot resonances $\mathbf{E}^{(\mathrm{FP})}$.

At this point, the crucial issue is what exactly governs the higher/lower degree of interference in eqs 5 and 6: basically, the far-field integral over solid angles in eq 5 which both electric field amplitudes in eq 6 contribute to. To shed light on it, we now plot the far-field pattern associated with all (Mie and FP) resonances. Mie-like far-field patterns 
are calculated numerically (SCUFF) to properly account for the finiteness of the NW. They resemble over a long interval of polar angles the Mie theory calculation for an infinite cylinder, ${ }^{6}$ being nearly identical at a far-field plane perpendicular to the nanorod axis, while expectedly breaking the translational invariance along the nanorod axis so that the angular pattern vanishes at its poles. These Mie-like patterns (shown in Figures 5a, 5d and 6a) are calculated within the corresponding broad Mie resonances, but at a fixed wavelength chosen so that no overlap occurs with the narrow FP resonances.

\section{Mie-like}

(a) $\mathrm{a}_{0}$

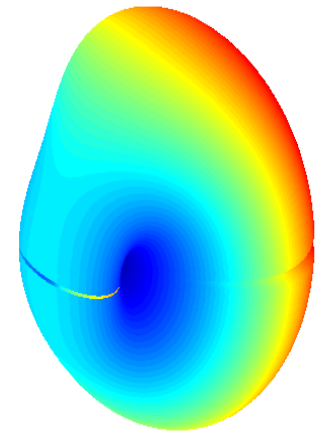

(d) $a_{1}$

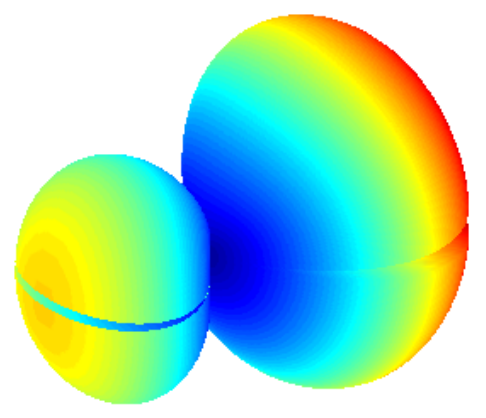

Fabry-Perot $\mathrm{TE}_{01}$

(b) $n=3$

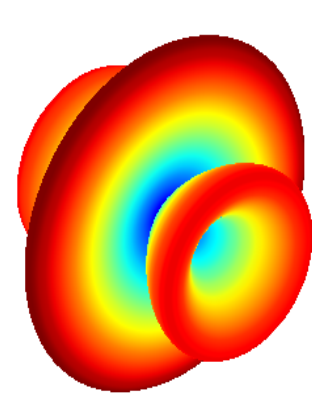

(e) $n=5$

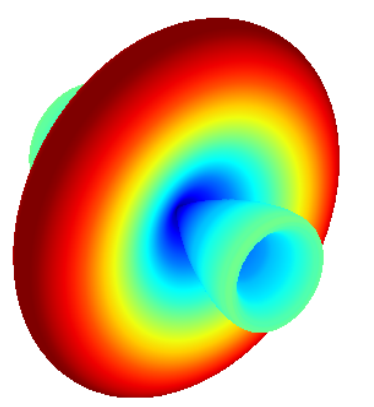

Total

(c)

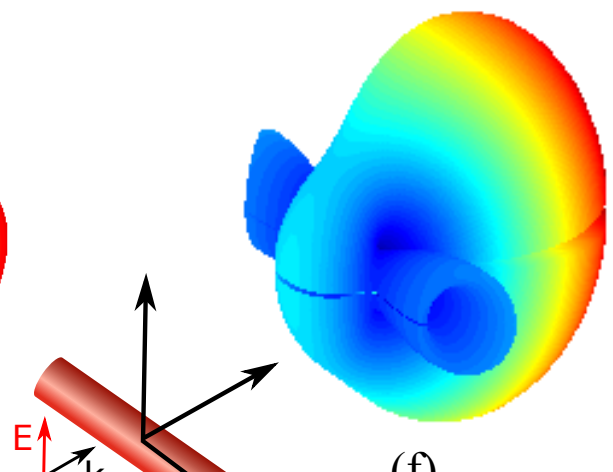

(f)

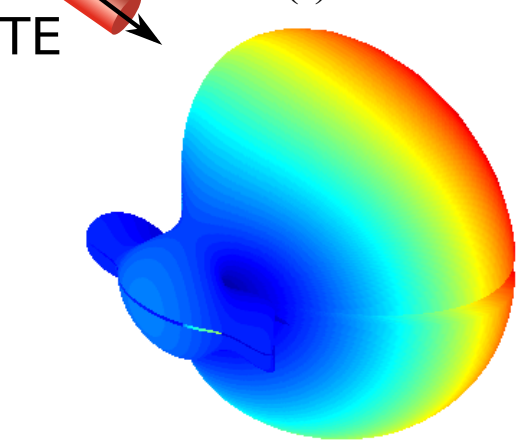

Figure 5: Far-field angular scattering patterns at normal incidence for TE polarization for a finite semiconductor ( $n=3.5$ ) nanorod of radius $R=80 \mathrm{~nm}$ and fixed length $L=600 \mathrm{~nm}$, calculated through: (a,c,d,f) SCUFF numerical calculations at the following wavelengths: (a) $\lambda=740 \mathrm{~nm}$ (isolated $a_{0}$ mode) and (d) $\lambda=490 \mathrm{~nm}$ (isolated $a_{1}$ mode); (c) $\lambda=635 \mathrm{~nm}$ and (f) $\lambda=525 \mathrm{~nm}$ at Fano resonance maxima. (b,e) 1D line current model (eqs 7 and 9) for the following odd $\mathrm{FP}$ resonances (see eq 1): $\mathrm{TE}_{01}^{(3)}$ at $\lambda=635 \mathrm{~nm}$, (e) $\mathrm{TE}_{01}^{(5)}$ at $\lambda=525$ nm. The inset depicts the scattering geometry.

Far-fields of Fabry-Perot resonances cannot be extracted from the numerical calcula- 
tions, due to the fact that the resulting patterns are dominated by the profiles of the background Mie resonance. To infer the far-field profile of such (isolated) FP resonances, we use the following expressions extrapolated from Refs., ${ }^{54,55}$ that account for the far-field emitted/scattered by a 1D line current. For transverse electric or magnetic modes with (magnetic or electric, respectively) polarization along the nanorod axis, the electric far field exhibits axial symmetry, so its only component is the polar one:

$$
E_{\theta}^{(\mathrm{FP})}=\frac{\imath k_{0} \eta_{0} S_{x y}}{4 \pi} \sin \theta \int_{-L / 2}^{L / 2} J(z) e^{-\imath k_{0} z \cos \theta} d z
$$

where $\eta_{0}$ stands for the vacuum impedance and $S_{x y}=\pi R^{2}$ is the geometrical cross section of the nanorod. For hybrid guided modes with non-transverse polarization, such as the $\mathrm{HE}_{11}$ mode, the far-field pattern can be written as follows:

$$
\begin{aligned}
& E_{\theta}^{(\mathrm{FP})}=\frac{\imath k_{0} \eta_{0} S_{x y}}{4 \pi} \cos \theta \sin \phi \int_{-L / 2}^{L / 2} J(z) e^{-\imath k_{0} z \cos \theta} d z, \\
& E_{\phi}^{(\mathrm{FP})}=\frac{\imath k_{0} \eta_{0} S_{x y}}{4 \pi} \cos \phi \int_{-L / 2}^{L / 2} J(z) e^{-\imath k_{0} z \cos \theta} d z .
\end{aligned}
$$

The 1D current density of the FP resonances at normal incidence is expressed in the following manner:

$$
J(z)=J_{\gamma} \frac{\cos k_{z}^{(\gamma)}(\omega) z}{\cos k_{z}^{(\gamma)}(\omega) L^{*} / 2}
$$

where $\gamma$ denotes the guided mode involved, $J_{\gamma}$ being an unknown constant depending on each mode. The above expression has been deduced from the $1 \mathrm{D}$ current used for metallic nanorods in Ref. ${ }^{46}$ at normal incidence, so that only odd FP resonant modes (see eq 1 for $l=1,3, \ldots)$ are accounted for, which suffices for our purpose.

We can now qualitatively determine the emergence of Fano line shapes at the corresponding FP resonances. The highly asymmetric Fano resonance for TE polarization at $\lambda \approx 635$ $\mathrm{nm}$ in Figure 2 results from the $\mathrm{TE}_{01}^{(3)} \mathrm{FP}$ mode and lies within the broad $a_{0}$ Mie resonance. 


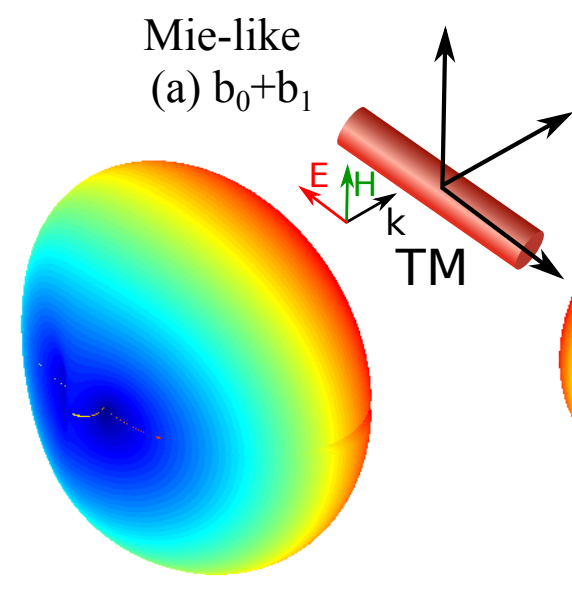

Fabry-Perot $\mathrm{HE}_{11}$

(b) $n=3$

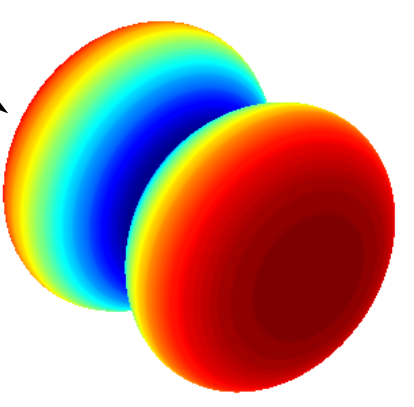

Total

(c)

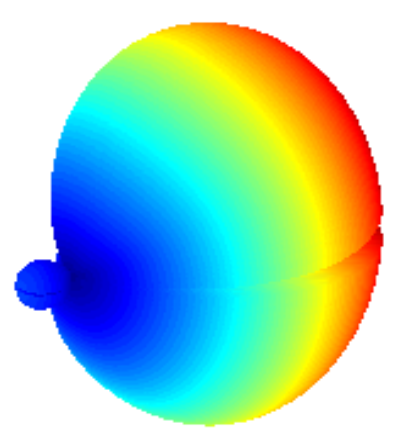

Figure 6: Far-field angular scattering patterns at normal incidence for TM polarization for a finite semiconductor $(n=3.5)$ nanorod of radius $R=80 \mathrm{~nm}$ and fixed length $L=500$ nm, calculated through: (a,c) SCUFF numerical calculations at the following wavelengths: (a) $\lambda=800 \mathrm{~nm}$ (isolated $b_{1}$ mode) and (c) $\lambda=670 \mathrm{~nm}$ at a Lorentz resonance. (b) 1D line current model (eqs 8 and 9) for the odd FP resonances (see eq 1) $\mathrm{HE}_{11}^{(3)}$ at $\lambda=670 \mathrm{~nm}$. The inset depicts the scattering geometry.

Both exhibit far-field patterns that strongly overlap (see Figures 5a and 5b), specially the large central ring of the FP pattern with the elliptical lobe of the Mie-like $a_{0}(\mathrm{eMD})$ at similar scattering angles, and indeed with analogous polarization (electric field perpendicular to the nanorod axis, rotating around it), which therefore interfere very strongly. Likewise, the other strong Fano resonance in Figure 2 at $\lambda \approx 525 \mathrm{~nm}$ is due to the strong interference of the $\mathrm{TE}_{01}^{(5)} \mathrm{FP}$ mode with the broad $a_{1}$ Mie resonance, both with overlapping far-field patterns: see Figures 5d and 5e, in particular the FP central ring with the dipole-like pattern with two lobes of the ED Mie resonance.

Conversely, the weakly asymmetric resonances for TM polarization at $\lambda \approx 640 \mathrm{~nm}$ in Figure 3 results from the narrow $\mathrm{HE}_{11}^{(3)} \mathrm{FP}$ mode at $\lambda \approx 670 \mathrm{~nm}$, overlying on a broad background with contributions from both $b_{0}$ and $b_{1}$ Mie resonances. Weak interference is expected since the corresponding far-field pattern of the FP mode, with two polar (yo-yolike) lobes, barely overlaps with the center lobe of the $b_{0}, b_{1}$ Mie-like far-field pattern (see Figures $6 \mathrm{a}$ and $6 \mathrm{~b})$. The same argument explains the nearly Lorentzian lineshape of the narrow $\mathrm{HE}_{11}^{(5)} \mathrm{FP}$ mode at $\lambda \approx 500 \mathrm{~nm}$, whose far field overlaps even less than the former 
FP mode with that of the background, stemming from the broad $b_{1}$ and (only slightly) $b_{2}$ Mie resonances (not shown here).

\section{Fano line shapes}

Based on the above physical arguments, we next propose a phenomenological expression the spectral dependence of the SCS that accounts for the Fano interference as follows:

$$
\begin{gathered}
\operatorname{SCS}^{\mathrm{TE}}(\omega)=\frac{4 L}{k}\left\{\alpha_{0}\left|a_{0}(\omega)\left[1+\sum_{i=1}^{\mathrm{FP}_{m}} \frac{\phi_{0 i}}{\nu_{i}+\imath}\right]\right|^{2}\right. \\
\left.+2 \sum_{m=1}^{\infty} \alpha_{m}\left|a_{m}(\omega)\left[1+\sum_{i=1}^{\mathrm{FP}_{m}} \frac{\phi_{m i}}{\nu_{i}+\imath}\right]\right|^{2}\right\} \\
\operatorname{SCS}^{\mathrm{TM}}(\omega)=\frac{4 L}{k}\left\{\beta_{0}\left|b_{0}(\omega)\left[1+\sum_{i=1}^{\mathrm{FP}_{m}} \frac{\phi_{0 i}}{\nu_{i}+\imath}\right]\right|^{2}\right. \\
\left.+2 \sum_{m=1}^{\infty} \beta_{m}\left|b_{m}(\omega)\left[1+\sum_{i=1}^{\mathrm{FP}_{m}} \frac{\phi_{m i}}{\nu_{i}+\imath}\right\}\right|^{2}\right]
\end{gathered}
$$

The above SCS is nothing but the Mie scattering efficiencies in eqs 4, converted to scattering cross sections by multiplying by a geometrical factor $2 R L$, allowing also for a constant factor $\alpha_{m}, \beta_{m}$ independent for each Mie contribution, to match the background of the numerical calculation (for the sake of completeness); FP resonances are included through complex Lorentzians, where $\nu_{i}$ is their reduced frequency

$$
\nu_{i}=\left(\omega-\omega_{i}\right) / \Gamma_{i},
$$

with frequency $\omega_{i}$ and halfwidth $\Gamma_{i}$. Every FP resonance is allowed to interfere with the $m$-th Mie contribution according to their $\phi_{m i}$ factor, which can indeed be complex.

On the other hand, we will also fit the resulting line shapes to a canonical Fano formula. ${ }^{38}$ However, the fact that the role of the continuum is played in Plasmonics by a broad resonance 


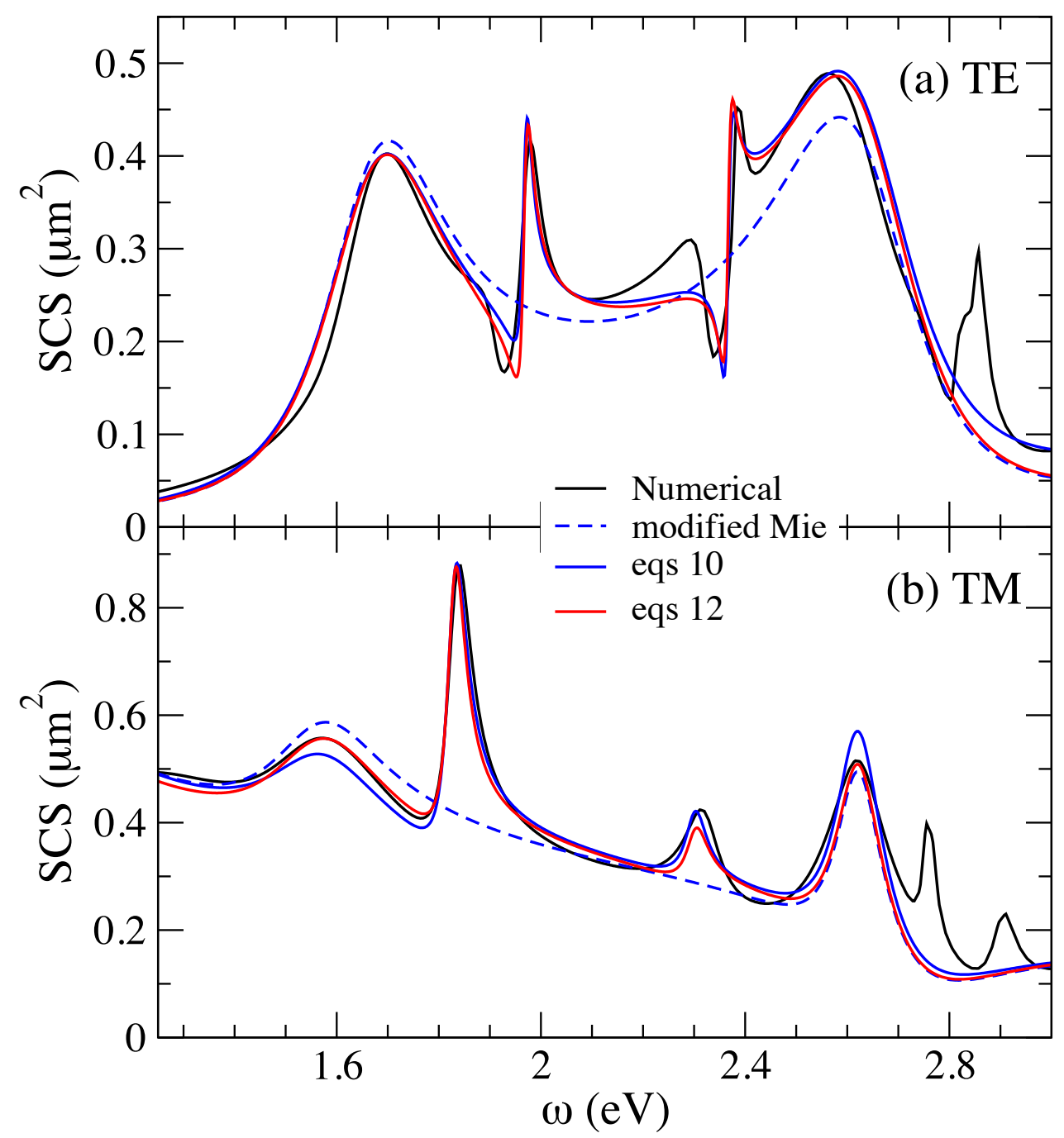

Figure 7: Spectral dependence of the scattering cross section $Q_{\text {sca }}$ at normal incidence for a finite semiconductor ( $n=3.5$ ) nanorod of radius $R=80 \mathrm{~nm}$ and fixed length $L$, calculated through eqs 10 (blue curves) and generalized Fano formula (eqs 12, red curves), both in turn including the Mie coefficients from eq 4, modified to properly account for the finite nanorod background (dashed blue curves) as described in the text. The exact (SCUFF) numerical calculation is also included for comparison (black curves). (a) TE polarization, $L=600 \mathrm{~nm}$. (b) TM polarization, $L=500 \mathrm{~nm}$. 
modifies the canonical Fano line shape in a peculiar manner, as pointed out in Refs. ${ }^{40,41}$ with different approaches. In our case, a few broad Mie resonances contribute to the background, rather than a single broad dipolar (plasmon) resonance. Therefore, we introduce a heuristic expression extending that of Ref. ${ }^{41}$ to account for the complex background scattered by semiconductor nanorods, as follows

$$
\begin{aligned}
& \operatorname{SCS}^{\mathrm{TE}}(\omega)=\frac{4 L}{k}\left[\alpha_{0}\left|a_{0}(\omega)\right|^{2}+2 \sum_{m=1}^{\infty} \alpha_{m}\left|a_{m}(\omega)\right|^{2}\right] \prod_{i=1}^{\mathrm{FP}} \frac{\left(\nu_{i}+q_{i}\right)^{2}+\delta_{i}}{\nu_{i}^{2}+1} ; \\
& \operatorname{SCS}^{\mathrm{TM}}(\omega)=\frac{4 L}{k}\left[\beta_{0}\left|b_{0}(\omega)\right|^{2}+2 \sum_{m=1}^{\infty} \beta_{m}\left|b_{m}(\omega)\right|^{2}\right] \prod_{i=1}^{\mathrm{FP}} \frac{\left(\nu_{i}+q_{i}\right)^{2}+\delta_{i}}{\nu_{i}^{2}+1}
\end{aligned}
$$

where $q_{i}, \delta_{i}$ are generalized Fano factors determining, respectively, the degree of asymmetry (as in the canonical Fano formula) and the contrast, the latter in turn related to intrinsic losses. $^{41}$

The resulting SCSs from our heuristic expression (eqs 10) and the generalized Fano formula (eqs 12), are shown in Figure 7 in the same cases as shown in Figures 2 and 3, but in the frequency domain to clearly reveal the asymmetric line shapes. The lowest-order Mie contributions considered in the calculation have been corrected in Figure 7 with the following factors

$$
\alpha_{0}=1.25, \alpha_{1}=1.03, \beta_{0}=1.65, \beta_{1}=0.44, \beta_{2}=1.3 .
$$

As expected, the impact of the finiteness of the nanorod (with regard to the infinite nanocylinder described by Mie theory) is much larger in TM polarization, since the electric field in the latter case is polarized along the nanorod axis. The total SCSs including FP resonances from either eqs 10 or eqs 12 exhibit a good agreement with the numerical simulations previously 
shown. The corresponding parameters in eqs 10 are:

$$
\begin{aligned}
\phi_{01}^{(\mathrm{TE})}=0.2-1.14 \imath, \phi_{02}^{(\mathrm{TE})} & =0.5+0.1 \imath, \phi_{1 i}^{(\mathrm{TE})}=\phi_{0 i}^{(\mathrm{TE})} e^{-\imath \pi / 4}, i=1,2 ; \\
\phi_{m 1}^{(\mathrm{TM})} & =1.7+0.6 \imath, \phi_{m 2}^{(\mathrm{TM})}=0.3+0.8 \imath, m=0,1 .
\end{aligned}
$$

And the generalized Fano factors used in eqs 12 are given by

$$
\begin{aligned}
& q_{1}^{(\mathrm{TE})}=0.53, \delta_{1}^{(\mathrm{TE})}=1.2, q_{2}^{(\mathrm{TE})}=0.44, \delta_{1}^{(\mathrm{TE})}=0.85 \\
& q_{1}^{(\mathrm{TM})}=0.3, \delta_{1}^{(\mathrm{TM})}=1.95, q_{2}^{(\mathrm{TM})}=0.08, \delta_{1}^{(\mathrm{TM})}=1.3 .
\end{aligned}
$$

All four FP Fano resonances are qualitatively (and nearly quantitatively) reproduced regardless of their degree of asymmetry. Particularly, the high asymmetry and contrast of the line shapes in TE polarization are accounted for through the fitting parameters in the following manner: large $\phi^{(\mathrm{TE})}$ factors (either nearly real or imaginary) in eqs 10; and large $q^{(\mathrm{TE})}$ and relatively small $\delta^{(\mathrm{TE})}$ factors, though non-negligible (for $\delta=0$ the resonance minimum would be zero), in eqs 12 . On the other hand, the weaker interference of the FP resonances in TM polarization is also manifested through: comparable real and imaginary parts in $\phi^{(\mathrm{TM})}$ in eqs 10 (weaker interference); much larger $\left|\phi^{(\mathrm{TM})}\right|$ for the stronger, lower

frequency FP resonance. And correspondingly, small Fano factors $q^{(\mathrm{TM})}$ and large $\delta^{(\mathrm{TM})}$ in eqs 12 that make the line shape slightly asymmetric and relatively large. Overall, this remarkable agreement firmly supports our physical interpretation relying on eqs 6, included heuristically in the scattered amplitude in eqs 10, and in the scattered intensity through the generalized Fano formula in eqs 12.

\section{Conclusions}

To summarize, we have investigated theoretically and numerically light scattering from finite semiconducting nanorods, with radius of the order of a hundred nanometers and lengths of 
about half micron, such that lowest-order Mie resonances coexist with Fabry-Perot resonances of lowest-order guided modes. The rich phenomenology associated to such variety of resonances has been studied, with the aim of identifying Fano resonances. Indeed, highly asymmetric Fano line shapes are found in TE polarization, with far more contrast and intensity than those previously reported for metallic nanorods; less asymmetric, but also very strong, Fano resonances are found in TM polarization. Furthermore, we have given a physical interpretation in terms of the interference of far-field angular patterns of broad Mie resonances with narrow FP modes, accounted for with a 1D line current model. In this regard also, a simple expression is given to quasi-analytically determine the scattering cross sections, in excellent agreement with the numerical simulations. A generalized Fano formula extended to account for a broad multi-resonant continuum is also presented. Both yield asymmetry factors consistent with our physical interpretation. The phenomenology here reported opens up a new venue for Fano resonance engineering in semiconductor nanorods (and in general in high-refractive index cylinders at any spectral regime), which might become even richer if more FP and/or Mie modes, and off-normal incidence are included. Our high contrast Fano resonances could be exploited in Nanophotonics and Sensing, and in general in a wide range of technologies, bearing in mind that future integration into new optical media will be largely eased since only all-dielectric nanofabrication (lossless GaP, crystalline silicon, or any other semiconductor not too lossy at the frequency of interest) is required.

\section{Methods}

Numerical calculations. Numerical simulations through SCUFF ${ }^{51,52}$ (open-source software package for analysis of electromagnetic scattering problems using the Method of Moments) have been carried out, in particular for scattering cross sections and far field angular patterns. In all cases, the (only) needed mesh file for the surface of the nanorod was obtained from that used as COMSOL finite cylinder with extra-fine mesh. 
Finite-element-method-based numerical simulations were performed using commercial software COMSOL Multiphysics v4.3b. The simulated space consisted of a circular cylinder of length $\mathrm{L}$ and diameter $\mathrm{D}$, representing the nanorod, and a cylinder of length $2 L$ and radii $0.5 L$ with its center coinciding with that of the nanowire. In the outer cylinder, an additional layer of thickness $0.2 \mathrm{~L}$ was defined. The nanorod domain was set to be a lossless semiconductor with $n=3.5$, with material constants taken from Palik's book, ${ }^{53}$ while the rest of the space was set to be air $\left(n_{\text {air }}=1\right)$. The outer cylindrical layer was defined as a perfectly matched layer (PML) to absorb all the outgoing radiation. The meshing was done with the program built-in algorithm, which creates a tetrahedral mesh. The mesh maximum element size (MES), which limits the maximum size of the edges of the tetrahedrons, was set to be $10 \mathrm{~nm}$ in the domain representing the wire and $120 \mathrm{~nm}$ for the rest of the domains. The maximum element growth rate was set to 1.3 in the wire domain, and 1.35 in all remaining domains. Direct PARDISO solver was used to solve the problem. Simulations needed typically $30 \mathrm{~GB}$ of memory and took about thirty minutes per wavelength on a 32 CPUs computing station.

\section{Acknowledgement}

This work has been supported by the Spanish "Ministerio de Economía y Competitividad" (projects LENSBEAM FIS2015-69295-C3-2-P and RedMETA TEC2015-69195-REDC, and FPU Fellowship), and CSIC (project NANOPHOTOPLAS, i-COOP LIGHT 2015CD0011).

\section{Supporting Information Available}

Numerical calculations of the SCS for the same nanorod dimensions used in Figures 2 and 3, but for a real semiconductor, GaP, with dielectric function from ref. ${ }^{53}$ This material is available free of charge via the Internet at http://pubs . acs.org/. 


\section{References}

(1) Kuznetsov, A. I.; Miroshnichenko, A. E.; Brongersma, M. L.; Kivshar, Y. S.; Luk'yanchuk, B. Optically resonant dielectric nanostructures. Science 2016, 354, $\operatorname{aag} 2472$.

(2) Kivshar, Y.; Miroshnichenko, A. Meta-Optics with Mie resonances. Optics and Photonics News 2017, 24-31.

(3) Evlyukhin, A. B.; Reinhardt, C.; Seidel, A.; Luk'Yanchuk, B. S.; Chichkov, B. N. Optical response features of Si-nanoparticle arrays. Phys. Rev. B 2010, 82, 045404.

(4) García-Etxarri, A.; Gómez-Medina, R.; Froufe-Pérez, L. S.; López, C.; Chantada, L.; Scheffold, F.; Aizpurua, J.; Nieto-Vesperinas, M.; Sáenz, J. J. Strong magnetic response of submicron Silicon particles in the infrared. Opt. Express 2011, 19, 4815-4826.

(5) Paniagua-Domínguez, R.; López-Tejeira, F.; Marqués, R.; Sánchez-Gil, J. A. Metallodielectric coreshell nanospheres as building blocks for optical three-dimensional isotropic negative-index metamaterials. New J. Phys. 2011, 13, 123017.

(6) Bohren, C. F.; Huffman, D. R. Absorption and scattering of light by small particles; Wiley, N.Y., 1998.

(7) Geffrin, J. M.; García-Cámara, B.; Gómez-Medina, R.; Albella, P.; Froufe-Pérez, L. S.; Eyraud, C.; Litman, A.; Vaillon, R.; González, F.; Nieto-Vesperinas, M. et al. Magnetic and electric coherence in forward- and back-scattered electromagnetic waves by a single dielectric subwavelength sphere. Nat. Commun. 2012, 3, 1171.

(8) Kuznetsov, A. I.; Miroshnichenko, A. E.; Fu, Y. H.; Zhang, J.; Luk'yanchuk, B. Magnetic light. Sci. Rep. 2012, 2, 492.

(9) Evlyukhin, A. B.; Novikov, S. M.; Zywietz, U.; Eriksen, R. L.; Reinhardt, C.; Bozhevol- 
nyi, S. I.; Chichkov, B. N. Demonstration of Magnetic Dipole Resonances of Dielectric Nanospheres in the Visible Region. Nano Lett. 2012, 12, 3749-3755.

(10) Caldarola, M.; Albella, P.; Cortés, E.; Rahmani, M.; Roschuk, T.; Grinblat, G.; Oulton, R. F.; Bragas, A. V.; Maier, S. A. Non-plasmonic nanoantennas for surface enhanced spectroscopies with ultra-low heat conversion. Nat. Commun. 2015, 6, 7915.

(11) Fu, Y. H.; Kuznetsov, A. I.; Miroshnichenko, A. E.; Yu, Y. F.; Luk'yanchuk, B. Directional visible light scattering by silicon nanoparticles. Nat. Commun. 2013, 4, 1527.

(12) Staude, I.; Miroshnichenko, A. E.; Decker, M.; Fofang, N. T.; Liu, S.; Gonzales, E.; Dominguez, J.; Luk, T. S.; Neshev, D. N.; Brener, I. et al. Tailoring Directional Scattering through Magnetic and Electric Resonances in Subwavelength Silicon Nanodisks. ACS Nano 2013, 7, 7824-7832.

(13) Luk'yanchuk, B. S.; Voshchinnikov, N. V.; Paniagua-Domínguez, R.; Kuznetsov, A. I. Optimum Forward Light Scattering by Spherical and Spheroidal Dielectric Nanoparticles with High Refractive Index. ACS Photonics 2015, 2, 993-999.

(14) Cambiasso, J.; Grinblat, G.; Li, Y.; Rakovich, A.; Cortés, E.; Maier, S. A. Bridging the Gap between Dielectric Nanophotonics and the Visible Regime with Effectively Lossless Gallium Phosphide Antennas. Nano Lett. 2017, 17, 1219-1225.

(15) Moitra, P.; Yang, Y.; Anderson, Z.; Kravchenko, I. I.; Briggs, D. P.; Valentine, J. Realization of an all-dielectric zero-index optical metamaterial. Nat. Photonics 2013, 7, 791-795.

(16) Paniagua-Domínguez, R.; Abujetas, D. R.; Sánchez-Gil, J. A. Ultra low-loss, isotropic optical negative-index metamaterial based on hybrid metal-semiconductor nanowires. Sci. Rep. 2013, 3, 1507. 
(17) Yang, Y.; Wang, W.; Moitra, P.; Kravchenko, I. I.; Briggs, D. P.; Valentine, J. Dielectric Meta-Reflectarray for Broadband Linear Polarization Conversion and Optical Vortex Generation. Nano Lett. 2014, 14, 1394-1399.

(18) Moitra, P.; Slovick, B. A.; Li, W.; Kravchencko, I. I.; Briggs, D. P.; Krishnamurthy, S.; Valentine, J. Large-Scale All-Dielectric Metamaterial Perfect Reflectors. ACS Photonics 2015, 2, 692-698.

(19) Paniagua-Domínguez, R.; Yu, Y. F.; Miroshnichenko, A. E.; Krivitsky, L. A.; Fu, Y. H.; Valuckas, V.; Gonzaga, L.; Toh, Y. T.; Kay, A. Y. S.; Luk'yanchuk, B. et al. Generalized Brewster effect in dielectric metasurfaces. Nat. Commun. 2016, 7, 10362.

(20) Kim, S. J.; Park, J.; Esfandyarpour, M.; Pecora, E. F.; Kik, P. G.; Brongersma, M. Superabsorbing, artificial metal films constructed from Semiconductor Nanoantennas. Nano Lett. 2016, 16, 3801-3808.

(21) Sell, D.; Yang, J.; Doshay, S.; Zhang, K.; Fan, J. A. Visible Light Metasurfaces Based on Single-Crystal Silicon. ACS Photonics 2016, 3, 1919-1925.

(22) Zhou, Z.; Li, J.; Su, R.; Yao, B.; Fang, H.; Li, K.; Zhou, L.; Liu, J.; Stellinga, D.; Reardon, C. P. et al. Efficient Silicon Metasurfaces for Visible Light. ACS Photonics 2017, 4, 544-551.

(23) Shcherbakov, M. R.; Neshev, D. N.; Hopkins, B.; Shorokhov, A. S.; Staude, I.; MelikGaykazyan, E. V.; Decker, M.; Ezhov, A. A.; Miroshnichenko, A. E.; Brener, I. et al. Enhanced Third-Harmonic Generation in Silicon Nanoparticles Driven by Magnetic Response. Nano Lett. 2014, 14, 6488-6492.

(24) Yang, Y.; Wang, W.; Boulesbaa, A.; Kravchenko, I. I.; Briggs, D. P.; Puretzky, A.; Geohegan, D.; Valentine, J. Nonlinear Fano-Resonant Dielectric Metasurfaces. Nano Lett. 2015, 15, 7388-7393. 
(25) Shcherbakov, M. R.; Vabishchevich, P. P.; Shorokhov, A. S.; Chong, K. E.; Choi, D.-Y.; Staude, I.; Miroshnichenko, A. E.; Neshev, D. N.; Fedyanin, A. A.; Kivshar, Y. S. Ultrafast All-Optical Switching with Magnetic Resonances in Nonlinear Dielectric Nanostructures. Nano Lett. 2015, 15, 6985-6990.

(26) Staude, I.; Khardikov, V. V.; Fofang, N. T.; Liu, S.; Decker, M.; Neshev, D. N.; Luk, T. S.; Brener, I.; Kivshar, Y. S. Shaping Photoluminescence Spectra with Magnetoelectric Resonances in All-Dielectric Nanoparticles. ACS Photonics 2015, 2, 172-177.

(27) Cao, L.; Fan, P.; Vasudev, A. P.; White, J. S.; Yu, Z.; Cai, W.; Schuller, J. A.; Fan, S.; Brongersma, M. L. Semiconductor Nanowire Optical Antenna Solar Absorbers. Nano Lett. 2010, 10, 439-445.

(28) Seo, K.; Wober, M.; Steinvurzel, P.; Schonbrun, E.; Dan, Y.; Ellenbogen, T.; Crozier, K. B. Multicolored Vertical Silicon Nanowires. Nano Lett. 2011, 11, 18511856.

(29) Grzela, G.; Paniagua-Domínguez, R.; Barten, T.; Fontana, Y.; Sánchez-Gil, J. A.; Gómez Rivas, J. Nanowire Antenna Emission. Nano Lett. 2012, 12, 5481-5486.

(30) Grzela, G.; Paniagua-Domínguez, R.; Barten, T.; van Dam, D.; Sánchez-Gil, J. A.; Rivas, J. G. Nanowire Antenna Absorption Probed with Time-Reversed Fourier Microscopy. Nano Lett. 2014, 14, 3227-3234.

(31) Brongersma, M. L.; Cui, Y.; Fan, S. Light management for photovoltaics using highindex nanostructures. Nat. Mater. 2014, 13, 451-460.

(32) Fan, P.; Yu, Z.; Fan, S.; Brongersma, M. L. Optical Fano resonance of an individual semiconductor nanostructure. Nat. Mater. 2014, 13, 471-475.

(33) Wu, C.; Arju, N.; Kelp, G.; Fan, J. A.; Dominguez, J.; Gonzales, E.; Tutuc, E.; 
Brener, I.; Shvets, G. Spectrally selective chiral silicon metasurfaces based on infrared Fano resonances. Nat. Commun. 2014, 5, 3892.

(34) Yang, Y.; Kravchenko, I. I.; Briggs, D. P.; Valentine, J. All dielectric metasurface analogue of electromagnetically induced transparency. Nat. Commun. 2014, 5, 5753.

(35) Hopkins, B.; Filonov, D. S.; Miroshnichenko, A. E.; Monticone, F.; Alù, A.; Kivshar, Y. S. Interplay of Magnetic Responses in All-Dielectric Oligomers To Realize Magnetic Fano Resonances. ACS Photonics 2015, 2, 724-729.

(36) Yan, J.; Liu, P.; Lin, Z.; Wang, H.; Chen, H.; Wang, C.; Yang, G. Directional Fano Resonance in a Silicon Nanosphere Dimer. ACS Nano 2015, 2968-2980.

(37) Yang, Y. Fano Interference of Electromagnetic Modes in Subwavelength Dielectric Nanocrosses. J. Phys. Chem. C 2016, 120, 21843-21849 .

(38) Miroshnichenko, A. E.; Flach, S.; Kivshar, Y. S. Fano resonances in nanoscale structures. Reviews of Modern Physics 2010, 82, 2257-2298.

(39) Luk'yanchuk, B.; Zheludev, N. I.; Maier, S. A.; Halas, N. J.; Nordlander, P.; Giessen, H.; Chong, C. T. The Fano resonance in plasmonic nanostructures and metamaterials. Nat. Mater. 2010, 9, 707-715.

(40) Giannini, V.; Francescato, Y.; Amrania, H.; Phillips, C. C.; Maier, S. A. Fano Resonances in Nanoscale Plasmonic Systems: A Parameter-Free Modeling Approach. Nano Lett. 2011, 11, 2835-2840.

(41) Gallinet, B.; Martin, O. J. F. Ab initio theory of Fano resonances in plasmonic nanostructures and metamaterials. Phys. Rev. B 2011, 83, 235427.

(42) Verellen, N.; Van Dorpe, P.; Huang, C.; Lodewijks, K.; Vandenbosch, G. A. E.; Lagae, L.; Moshchalkov, V. V. Plasmon Line Shaping Using Nanocrosses for High Sensitivity Localized Surface Plasmon Resonance Sensing. Nano Lett. 2011, 11, 391-397. 
(43) Chen, H.; Shao, L.; Ming, T.; Woo, K. C.; Man, Y. C.; Wang, J.; Lin, H.-Q.; Al, C. E. T. Observation of the Fano Resonance in Gold Nanorods Supported on High-DielectricConstant Substrates. Nano Lett. 2011, 5, 6754-6763.

(44) López-Tejeira, F.; Paniagua-Domínguez, R.; Rodríguez-Oliveros, R.; Sánchez-Gil, J. A. Fano-like interference of plasmon resonances at a single rod-shaped nanoantenna. New J. Phys. 2012, 14, 023035 .

(45) López-Tejeira, F.; Paniagua-Domínguez, R.; Sánchez-Gil, J. A. High-Performance Nanosensors Based on Plasmonic Fano-like Interference: Probing Refractive Index with nanorice and nanobelts. ACS Nano 2012, 6, 8989-8996.

(46) Verellen, N.; López-Tejeira, F.; Paniagua-Domínguez, R.; Vercruysse, D.; Denkova, D.; Lagae, L.; Van Dorpe, P.; Moshchalkov, V. V.; Sánchez-Gil, J. A. Mode ParityControlled Fano- and Lorentz-like Line Shapes Arising in Plasmonic Nanorods. Nano Lett. 2014, 14, 2322-2329.

(47) Bronstrup, G.; Jahr, N.; Leiterer, C.; Csaki, A.; Fritzsche, W.; Christiansen, S. Optical Properties of Individual Silicon Nanowires for Photonic Devices. ACS Nano 2010, 4 , $7113-7122$.

(48) Ee, H.-S.; Kang, J.-H.; Brongersma, M. L.; Seo, M.-K. Shape-Dependent Light Scattering Properties of Subwavelength Silicon Nanoblocks. Nano Lett. 2015, 15, 1759-1765.

(49) Landreman, P. E.; Chalabi, H.; Park, J.; Brongersma, M. L. Fabry-Perot description for Mie resonances of rectangular dielectric nanowire optical resonators. Opt. Express 2016, 24, 29760-29772.

(50) Traviss, D. J.; Schmidt, M. K.; Aizpurua, J.; Muskens, O. L. Antenna resonances in low aspect ratio semiconductor nanowires. Opt. Express 2015, 23, 22771-22787. 
(51) Homer Reid, M.; Johnson, S. Efficient Computation of Power, Force, and Torque in BEM Scattering Calculations. arXiv:1307.2966

(52) M. T. H. Reid. http://homerreid.com/scuffEM

(53) Palik, E. D. Handbook of Optical Constants of Solids; Academic Press, 1998; Vol. 3; p 900.

(54) Paniagua-Domínguez, R.; Grzela, G.; Rivas, J. G.; Sánchez-Gil, J. A. Enhanced and directional emission of semiconductor nanowires tailored through leaky/guided modes. Nanoscale 2013, 5, 10582-10590.

(55) Abujetas, D. R.; Sánchez-Gil, J. A.; Paniagua-Domínguez, R. Unraveling the Janus role of Mie resonances and leaky/guided modes in semiconductor nanowire absorption for enhanced light harvesting Leaky/guided modes versus Mie resonances. ACS Photonics 2015, 2, 921-929. 


\section{Graphical TOC Entry}

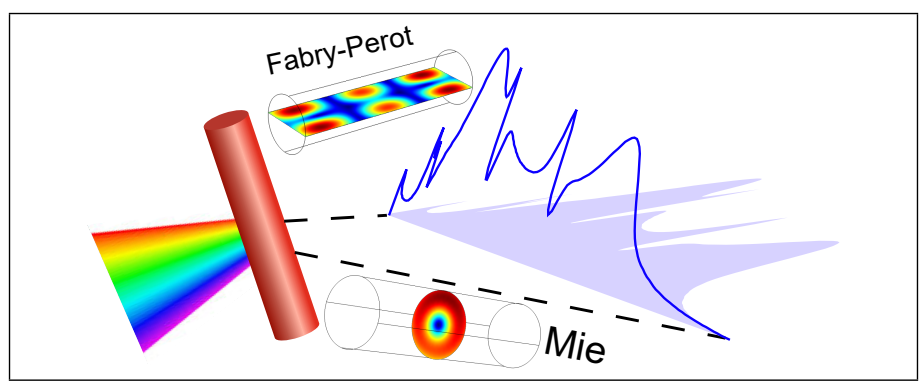

